\title{
UAV FOR GEODATA ACQUISITION IN AGRICULTUREAL AND FORESTAL APPLICATIONS
}

\author{
P. Reidelstürz ${ }^{\mathrm{a}, *}$, L. Schrenk ${ }^{\text {b }}$, W. Littmann ${ }^{.}$ \\ ${ }^{a}$ Hochschule Deggendorf, Technologie Campus Freyung, D-94078 Freyung, zugleich: \\ ext. Mitarbeiter am Institut für Robotik und Mechatronik, DLR-Oberpfaffenhofen, D- 82234 Wessling \\ patrick.reidelstuerz@fh-deggendorf.de \\ zuvor: Institut für Flugsysteme, Uni Bundeswehr, D-85577Neubiberg/MünchenD

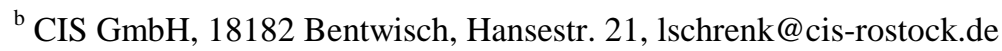 \\ ${ }^{c}$ ETL e.V. 18128 Bentwisch, Hansestr 21, info@etlev.de
}

Commission I, WG I/V

KEY WORDS: UAV, Autopilot, Flight Planning Tool, Data Processing, Precision Farming, Photogrammetry, Biomass Diversity, NDVI, diversity of water supply, Bark Beetle Detection in green attack phase;

\begin{abstract}
:
In the field of precision-farming research, solutions are worked out to combine ecological and economical requirements in a harmonic way. Integrating hightech in agricultural machinery, natural differences in the fields (biodiversity) can be detected and considered to economize agricultural resources and to give respect to natural ecological variability at the same time. Using precision farming resources, machining - and labour time can be economized, productivness can be improved, environmental burden can be discharged and documentation of production processes can be improved.

To realize precision farming it is essential to make contemporary large scale data of the biodiversity in the field available. In the last years effectual traktor based equipment for real time precision farming applications was developed. Using remote sensing, biomass diversity of the field can be considered while applicating operating ressources economicly.
\end{abstract}

Because these large scale data aquisition depends on expensive tractor based inspections, capable Unmanned Aerial Vehicles (UAVs) could complement or in special situations even replace such tractor based data aquisition needed for the realization of precision farming strategies.

The specific advantages and application slots of UAVs seems to be ideal for the usage in the field of precision farming. For example the size of even large agricultural fields in germany can be managed even by smaller UAVs. Data can be captured spontaneously, promptly, in large scale, with less respect of weather conditions. In agricultural regions UAV flights can be arranged in visual range as actually the legislator requires in germany, especially because the use of autopilotsystems in fact is nessecary to assure regular area-wide data without gaps but not to fly in non-visible regions. Also a minimized risk of hazard is given, flying UAVs over deserted agricultural areas.

In a first stage CIS GmbH cooperated with „Institute For Flightsystems“ of the University of German Armed Forces in Neubiberg/Munich and the well-established precision farming company „Konsultationszentrum Liepen“ to develop an applicable UAV for precision farming purposes. Currently Cis $\mathrm{GmbH}$ and Technologie Campus Freyung, with intense contact to the „flying robot"- team of DLR Oberpfaffenhofen, collaborate to optimize the existing UAV and to extend the applications from data aquisition for biomass diversity up to detect the water supply situation in agricultural fields, to support pest management systems as much as to check the possibilities to detect bark beetle attacks in european spruce in an early stage of attack (green attack phase) by constructing and integrating further payload modules with different sensors in the existing UAV airframe. Also effective data processing workflows are to be worked out.

Actually in the existing UAV autopilotsystem „piccolo“ (cloudcaptech) is integrated and also a replaceable payload module is available, carrying a VIS and a NIR camera to calculate maps of NDVI diversity as indicator of biomass diversity. Further modules with a 6 channel multispectral still camera and with a spectrometer are planned.

The airframe's wingspan is about $3,45 \mathrm{~m}$ weighting $4.2 \mathrm{~kg}$, ready to fly. The hand launchable UAV can start from any place in agricultural regions. The wing is configured with flaps, allowing steep approaches and short landings using a „butterfly“ brake configuration. In spite of the lightweight configuration the UAV yet proves its worth under windy baltic wether situations by collecting regular sharp images of fields under wind speed up to 15m/s (Beaufort 6 -7).

In further projects the development of further payload modules and a user friendly flight planning tool is scheduled considering different payload - and airframe requirements for different precision farming purposes and forest applications. Data processing and

\footnotetext{
* Corresponding author.
} 
workflow will be optimized. Cooperation with further partners to establish UAV systems in agricultural, forest and geodata aquisition is desired.

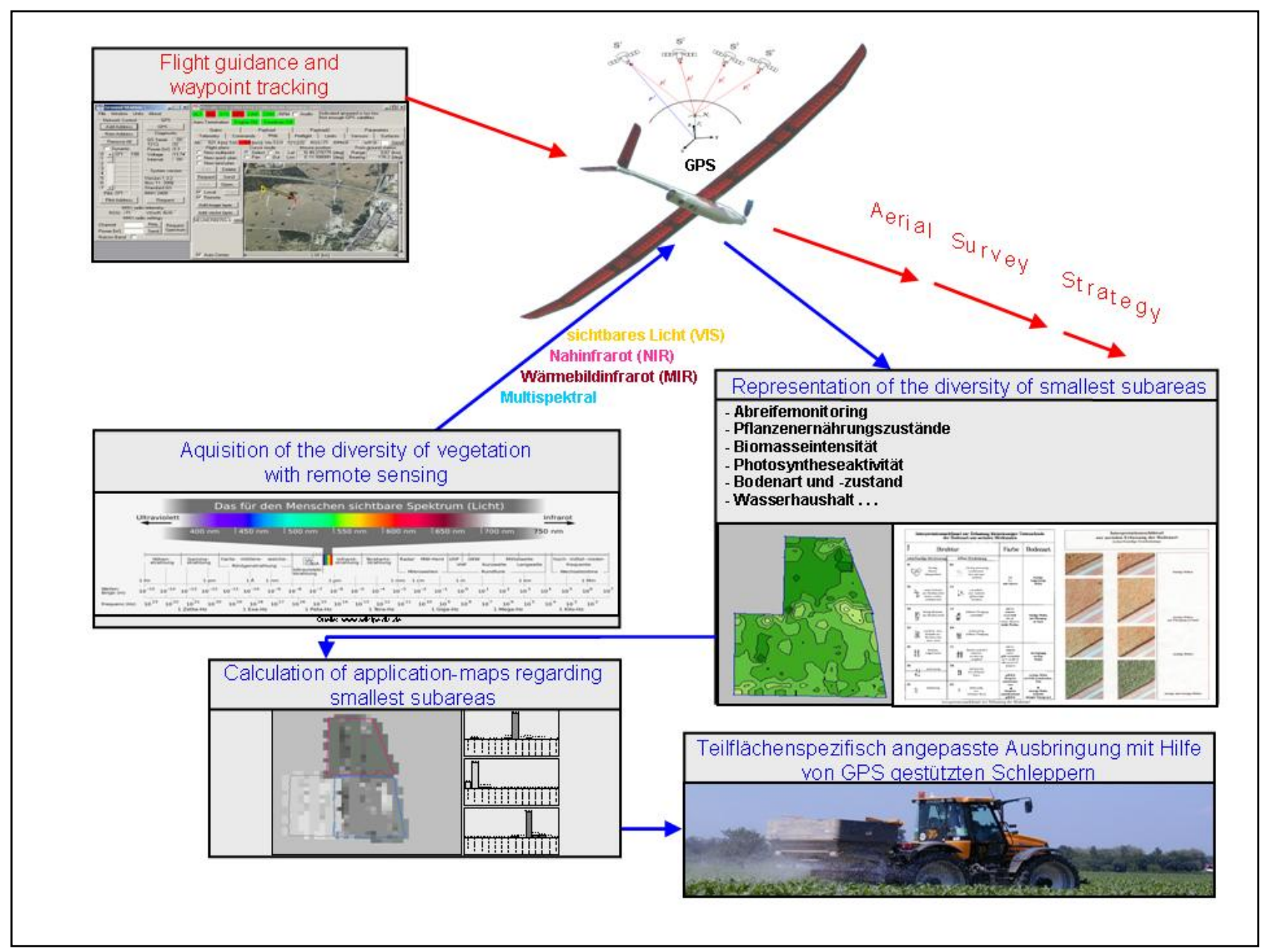

Figure 1: UAV with exchangeable payload module for data acquisition in precision farming, forestry and geoinformation 